\title{
Loss of Runt-related transcription factor 3 induces resistance to 5-fluorouracil and cisplatin in hepatocellular carcinoma
}

\author{
JUNRO KATAOKA $^{1}$, HIDENORI SHIRAHA ${ }^{1}$, SHIGERU HORIGUCHI ${ }^{1}$, HIROAKI SAWAHARA $^{1}$, \\ DAISUKE UCHIDA $^{1}$, TERUYA NAGAHARA ${ }^{1}$, MASAYA IWAMURO ${ }^{1}$, HIROKI MORIMOTO ${ }^{1}$, \\ YASUTO TAKEUCHI ${ }^{1}$, KENJI KUWAKI $^{1}$, HIDEKI ONISHI ${ }^{1}$, SHINICHIRO NAKAMURA ${ }^{1}$, \\ AKINOBU TAKAKI ${ }^{1}$, KAZUHIRO NOUSO ${ }^{1}$, TAKAHITO YAGI ${ }^{2}$, \\ KAZUHIDE YAMAMOTO ${ }^{3}$ and HIROYUKI OKADA ${ }^{1}$
}

\begin{abstract}
Departments of ${ }^{1}$ Gastroenterology and Hepatology, and ${ }^{2}$ Gastroenterological Surgery, Transplant and Surgical Oncology, Okayama University Graduate School of Medicine, Dentistry and Pharmaceutical Sciences, Okayama 700-8558;

${ }^{3}$ Department of Gastroenterology, Okayama Saiseikai General Hospital, Okayama 700-8511, Japan
\end{abstract}

Received November 11, 2015; Accepted December 18, 2015

DOI: $10.3892 /$ or.2016.4681

\begin{abstract}
Runt-related transcription factor 3 (RUNX3) is known to function as a tumor suppressor in gastric cancer and other types of cancers, including hepatocellular carcinoma (HCC). However, its role has not been fully elucidated. In the present study, we aimed to evaluate the role of RUNX3 in HCC. We used the human HCC cell lines Hep3B, Huh7 and HLF; RUNX3 cDNA was introduced into Hep3B and Huh7 cells, which were negative for endogenous RUNX3 expression, and RUNX3 siRNA was transfected into HLF cells, which were positive for endogenous RUNX3. We analyzed the expression of RUNX3 and multidrug resistance-associated protein (MRP) by immunoblotting. MTT assays were used to determine the effects of RUNX3 expression on 5-fluorouracil (5-FU) and cisplatin (CDDP) sensitivity. Finally, $23 \mathrm{HCC}$ specimens resected from patients with $\mathrm{HCC}$ at Okayama
\end{abstract}

Correspondence to: Dr Hidenori Shiraha, Department of Gastroenterology and Hepatology, Okayama University Graduate School of Medicine, Dentistry and Pharmaceutical Sciences, 2-5-1 Shikata-cho, Kita-ku, Okayama 700-8558, Japan

E-mail: hshiraha@okayama-u.ac.jp

Abbreviations: ABC, ATP-binding cassette; ABCC, ATP-binding cassette subfamily $\mathrm{C}$; $\mathrm{CAT}$, chloramphenicol acetyltransferase; cDNA, complementary DNA; CDDP, cisplatin; EMT, epithelial-mesenchymal transition; FBS, fetal bovine serum; 5-FU, 5-fluorouracil; HCC, hepatocellular carcinoma; MRP, multidrug resistance-associated protein; MTT, 3-(4,5-dimethylthiazol-2-yl)-2,5-diphenyltetrazolium bromide; RUNX3, Runt-related transcription factor 3; SDS, sodium dodecyl sulfate; siRNA, small interfering RNA; TBS-T, Tris-buffered saline with Tween-20; TGF- $\beta$, transforming growth factor- $\beta$

Key words: Runt-related transcription factor 3, hepatocellular carcinoma, chemoresistance, multidrug resistance-associated protein, 5-fluorouracil, cisplatin
University Hospital were analyzed, and correlations among immunohistochemical expression of RUNX3 protein and MRP protein were evaluated in these specimens. Exogenous RUNX3 expression reduced the expression of MRP1, MRP2, MRP3 and MRP5 in the RUNX3-negative cells, whereas knockdown of RUNX3 in the HLF cells stimulated the expression of these MRPs. An inverse correlation between RUNX3 and MRP expression was observed in the HCC tissues. Importantly, loss of RUNX3 expression contributed to 5-FU and CDDP resistance by inducing MRP expression. These data have important implications in the study of chemotherapy resistance in $\mathrm{HCC}$.

\section{Introduction}

Hepatocellular carcinoma (HCC) is the sixth most common cancer and the third most frequent cause of cancer-related mortality worldwide $(1,2)$. Although patients with early-stage HCC can receive curable treatment, the prognosis of patients with advanced disease is relatively poor. Drug resistance contributes to the poor prognosis in patients with advanced $\mathrm{HCC}$, and elucidation of the molecular mechanisms underlying drug resistance would facilitate the development of more effective therapeutic strategies $(3,4)$.

Drug efflux is a major molecular mechanism thought to affect drug resistance in patients with HCC. Increased drug efflux can decrease the accumulation of anticancer drugs (5). Multidrug resistance proteins (MRPs) are members of the ATP-binding cassette (ABC) transporter superfamily and are involved in drug efflux (6), contributing to the drug resistance observed in patients with HCC (5). Notably, MRPs are upregulated in 5-fluorouracil (5-FU)-resistant cancer cells, and MRP5 expression has been shown to influence 5-FU resistance in pancreatic carcinoma cells $(7,8)$. Furthermore, various studies have shown that there is a significant association between MRPs and 5-FU sensitivity (9-12). Drug resistance to cisplatin (CDDP), another common anticancer agent used for HCC chemotherapy (13-16), is also mediated by MRPs (17-20). 
Human Runt-related transcription factor 3 (RUNX3), a tumor suppressor expressed in gastric cancers (21), regulates cell growth and apoptosis as a downstream effector of transforming growth factor- $\beta$ (TGF- $\beta$ ) signaling (22). RUNX3 was originally reported as a tumor suppressor in gastric cancer (21) and has been shown to function as a tumor suppressor in HCC as well $(23,24)$. We and other researchers have reported that the protein and mRNA expression of RUNX3 are decreased in HCC and that loss of RUNX3 expression induces various effects in HCC, including prevention of apoptosis, induction of cancer stem cell-like changes, and promotion of the epithelialmesenchymal transition (EMT) $(24,25)$. Moreover, RUNX3 has been shown to regulate MRPs in pancreatic cancer (26). However, the mechanisms through which RUNX3 and MRPs may mediate drug resistance in HCC are not completely understood.

Therefore, in the present study, we assessed the relationship between RUNX3 and MRP expression in HCC. We also evaluated the effects of RUNX3 re-expression on drug resistance to 5-FU and CDDP in HCC.

\section{Materials and methods}

Cell lines and cell culture. The human HCC cell lines Hep3B, Huh7 and HLF were obtained from the American Type Culture Collection (ATCC; Manassas, VA, USA). The cells were maintained in Dulbecco's modified Eagle's medium (DMEM; Invitrogen, Carlsbad, CA, USA), supplemented with $10 \%$ heat-inactivated fetal bovine serum (FBS), $1 \%$ non-essential amino acids, $1 \%$ sodium pyruvate and $1 \%$ penicillin/streptomycin solution (all from Sigma, St. Louis, MO, USA). The cells were cultured at $37^{\circ} \mathrm{C}$ in an atmosphere containing $5 \%$ $\mathrm{CO}_{2}$ and $95 \%$ air. Cell growth was halted at subconfluency under restricted serum conditions with $0.1 \%$ dialyzed FBS for $36 \mathrm{~h}$ before the experiments, if needed.

Ectopic RUNX3 expression. A human RUNX3 construct was obtained by reverse transcription-polymerase chain reaction (RT-PCR)-based cloning of the gene from normal human hepatocytes (Sanko Junyaku, Co., Ltd., Tokyo, Japan). Human RUNX3 and/or chloramphenicol acetyltransferase (CAT; mock) constructs were transfected into Hep3B, Huh7 and HLF cells using FuGENE 6 transfection reagent (Roche Diagnostics, Basel, Switzerland). At least two independent transfections were performed for each cell line. The cells were incubated under serum-starved conditions for $24 \mathrm{~h}$ and used for the following experiments.

Immunoblot analysis. Cells were plated in 6-well plastic tissue culture dishes and grown to confluency. The cells were washed twice with cold phosphate-buffered saline (PBS) and lysed in $150 \mu \mathrm{l}$ of sample buffer [100 mNM Tris- $\mathrm{HCl}(\mathrm{pH} 6.8), 10 \%$ glycerol, $4 \%$ sodium dodecyl sulfate (SDS), $1 \%$ bromophenol blue and $10 \% \beta$-mercaptoethanol]. The samples were resolved by sodium dodecyl sulfate (SDS)-polyacrylamide gel electrophoresis and transferred to Immobilon-P polyvinylidene difluoride membranes (Millipore Corporation, Bedford, MA, USA). The membranes were blocked using Tris-buffered saline with Tween-20 (TBS-T; Sigma) containing 5\% bovine serum albumin for $1 \mathrm{~h}$. The membranes were then incubated with antibodies against RUNX3, MRP1 and MRP2 (all from Abcam, Cambridge, MA), MRP3 (Sigma), MRP5 (Abcam), and $\beta$-actin (Sigma) overnight at $4^{\circ} \mathrm{C}$. The membranes were washed 3 times with TBS-T and probed with horseradish peroxidase-conjugated secondary antibodies before being developed with an enhanced chemiluminescence western blotting detection system (Amersham Biosciences, Piscataway, NJ, USA).

MTT assay. Cell proliferation was assessed using 3-(4,5dimethylthiazol-2-yl)-2,5-diphenyl tetrazolium bromide (MTT) assays. Briefly, cells were grown in 96-well plastic tissue culture dishes at a density of $1 \times 10^{4}$ cells $/ \mathrm{ml}$. After $24 \mathrm{~h}$ of quiescence, cells were cultured for the indicated period with or without $10 \%$ FBS. Then, $10 \mu \mathrm{l}$ of MTT $(5 \mathrm{mg} / \mathrm{ml}$ in PBS) was added to each well, and the cells were incubated for an additional $4 \mathrm{~h}$ at $37^{\circ} \mathrm{C}$. The purple-blue formazan precipitate was dissolved in $100 \mu 1$ of dimethyl sulfoxide (DMSO). Mitochondrial activity, which reflected cell viability, was evaluated by measuring the optical density at $570 \mathrm{~nm}$ on a microplate reader (Bio-Rad, Hercules, CA, USA).

To evaluate 5-FU and CDDP resistance, the cells were treated with various concentrations of 5-FU and/or CDDP for $72 \mathrm{~h}$, and MTT assays were then performed as described above.

Gene silencing of RUNX3 with small interfering RNA (siRNA). RUNX3-expressing Hep3B and Huh7 cells were transfected with either scrambled negative control siRNA or RUNX3 siRNA (Applied Biosystems, Foster City, CA, USA) using RNAiFect transfection reagent (Qiagen, Hilden, Germany). The cells were incubated for $24 \mathrm{~h}$, and then serum starved for $48 \mathrm{~h}$. MTT assays were then performed.

HCC tissues and immunohistochemistry. A group of 23 patients [18 men (age range, 52-78 years; average age 65.1 years) and five women (age range, 55-74 years; average age, 65.8 years)] were included in the present study. Resected HCC tissues were obtained after receiving written informed consent that adhered to the stringent ethical criteria of the Okayama University Graduate School of Medicine, Dentistry and Pharmaceutical Sciences. Immunohistochemistry was performed on formalin-fixed paraffin-embedded sections prepared from the resected tissue. Sections were dewaxed and dehydrated; after rehydration, endogenous peroxidase activity was blocked for $30 \mathrm{~min}$ in a methanol solution containing $0.3 \%$ hydrogen peroxide. Following antigen retrieval in citrate buffer, the sections were blocked again overnight at $4^{\circ} \mathrm{C}$. The sections were probed with anti-RUNX3 mouse monoclonal antibodies (ab40278), anti-MRP1 monoclonal antibodies (ab32574), anti-MRP2 monoclonal antibodies (ab15603) (all from Abcam), anti-MRP3 monoclonal antibodies (M6567; Sigma) and anti-MRP5 monoclonal antibodies (ab24107; Abcam). The primary antibody was detected using a biotinylated anti-rabbit antibody or a biotinylated anti-mouse antibody (both from Dako Japan). The signal was amplified by avidin-biotin complex formation and was developed with diaminobenzidine, followed by dehydration in alcohol and xylene. The sections were then mounted and scored for RUNX3, MRP1, MRP2, MRP3 and MRP5 using a four-point 


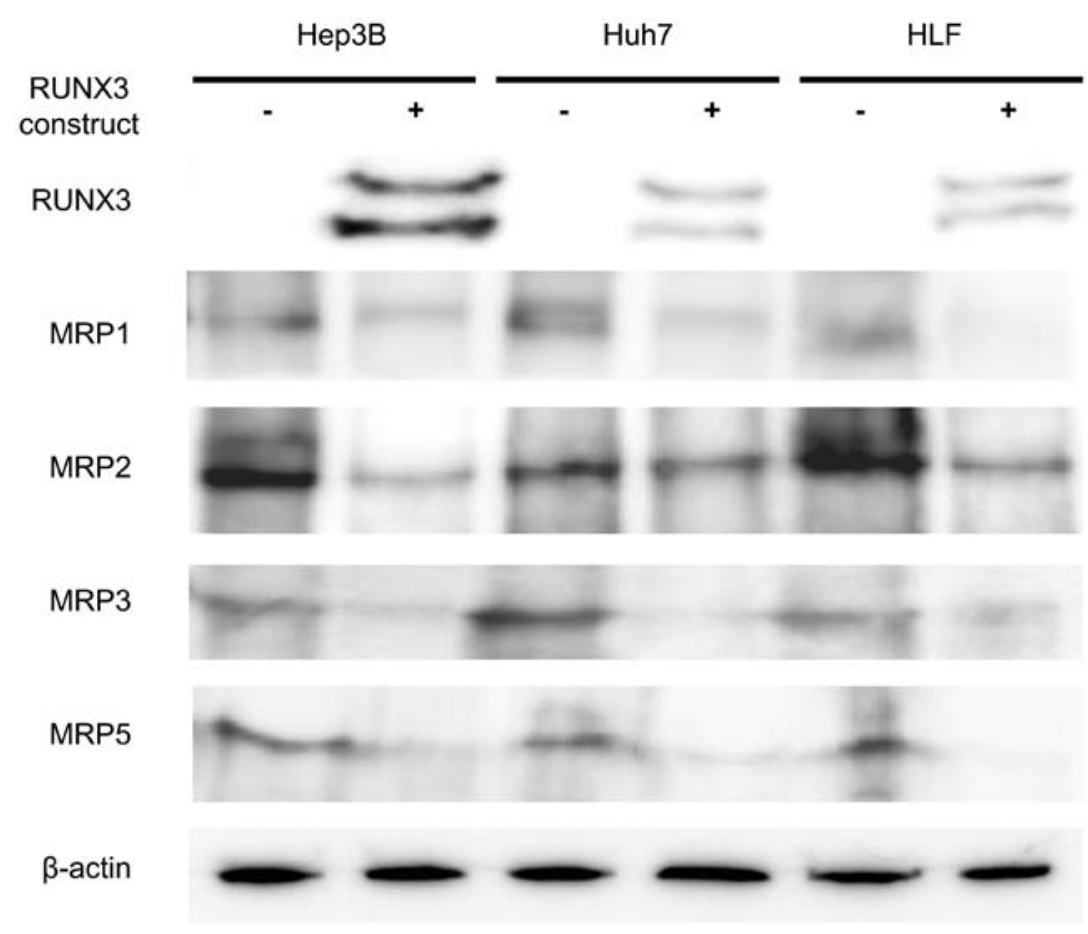

Figure 1. Immunoblot analysis. Eukaryotic expression constructs for CAT (mock) and RUNX3 were introduced into Hep3B, Huh7 and HLF cells. Cell lysates were collected $48 \mathrm{~h}$ after transfection. Immunoblot analysis was performed using antibodies against RUNX3, MRP1, MRP2, MRP3, MRP5 and $\beta$-actin. Immunoblotting for $\beta$-actin was used to verify equal loading of cellular proteins. Representative blots of more than 3 independent experiments are shown.

scale: 0 , negative; 1 , weak signal; 2 , intermediate signal; and 3 , strong signal. All sections were scored independently by two observers without prior knowledge of the clinical background. All discrepancies in scoring were reviewed, and a consensus was reached. Statistical analyses were performed using JMP software (SAS Institute, Inc., Cary, NC, USA).

Gene expression profiling analysis in human HCC. To further investigate correlations between RUNX3 and MRP expression in HCC, publicly available HCC data sets were evaluated using Oncomine (http://www.oncomine.org). In brief, mRNA expression profiles for RUNX3, MRP1, MRP2, MRP3 and MRP5 were evaluated using RUNX3/106_at, RUNX3/204197_s_at, RUNX3/204198_s_at, MRP1/34384_at, MRP2/206155_at, MRP3/209641_s_at, and MRP5/209380_s_at, respectively, in HCCs from the human liver data sets. Statistical analyses were performed using JMP software.

\section{Results}

Ectopic RUNX3 expression reduces MRP expression in HCC cell lines. Ectopic RUNX3 protein expression was regulated in $3 \mathrm{HCC}$ cell lines exhibiting positive or negative endogenous RUNX3 expression (Hep3B, Huh7 and HLF cells) (24). RUNX3 protein expression was detected by immunoblot analysis after transfection with the RUNX3 plasmid (Fig. 1). Hep3B, Huh7 and HLF cells expressed MRP1, MRP2, MRP3 and MRP5. Ectopic RUNX3 protein expression generally decreased MRP expression levels in all 3 cell lines. Expression of MRP1, MRP2, MRP3 and MRP5 in the RUNX3-expressing Hep3B, Huh7 and HLF cells was weaker than that in the control CAT-expressing cells (Fig. 1).
Ectopic RUNX3 protein expression suppresses cell growth and increases 5-FU and CDDP sensitivity. Ectopic RUNX3 expression suppressed cell growth in the Hep3B and Huh7 cells compared with that in the control Hep3B and Huh7 cells at 3 days after transfection (Fig. 2). Next, we analyzed the effects of RUNX3 on chemosensitivity in the RUNX3- or CAT (mock)-transfected Hep3B and Huh7 cells. RUNX3 expression enhanced 5-FU sensitivity in both cell lines; the $50 \%$ inhibitory concentration $\left(\mathrm{IC}_{50}\right)$ of 5-FU decreased from 8.16 to $4.84 \mathrm{nM}$ and from 9.81 to $4.76 \mathrm{nM}$ in the Hep3B and Huh7 cells, respectively (Fig. 2A). RUNX3 expression also enhanced CDDP sensitivity in both cell lines; the $\mathrm{IC}_{50}$ of CDDP decreased from 6.76 to $4.58 \mathrm{nM}$ and from 9.28 to $4.57 \mathrm{nM}$ in the Hep3B and Huh7 cells, respectively (Fig. 2B).

$M R P$ expression is inversely correlated with RUNX3 expression in human HCC tissues. Twenty-three HCC tissue samples were available for comparison of MRP and RUNX3 protein expression by immunohistochemistry. Representative images of tissues with different levels of MRP1 expression, i.e., negative (score 0), weak signal (score 1), intermediate signal (score 2) and strong signal (score 3), are shown in Fig. 3A-D. Correlation analysis showed that high MRP expression scores were generally observed in tissues with low RUNX3 expression (Fig. 4).

Gene expression profiling analysis in human HCC. Lastly, we evaluated the effects of ectopic RUNX3 expression on MRPs in order to elucidate the mechanisms underlying RUNX3 expression-induced chemosensitivity. We analyzed Oncomine data sets to examine the correlation between the expression of RUNX3 and MRPs. The results revealed that RUNX3 mRNA 
A

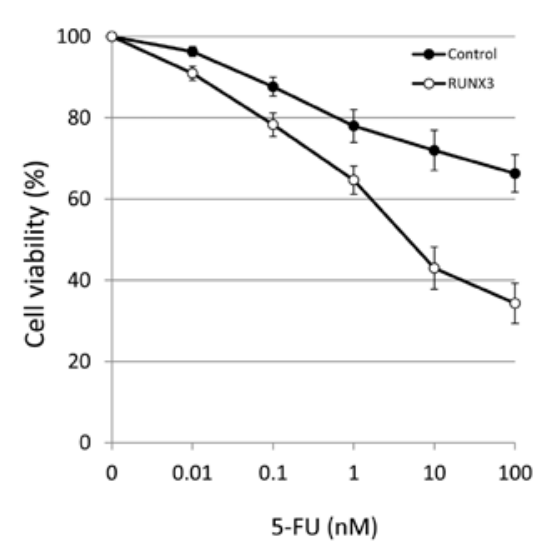

B

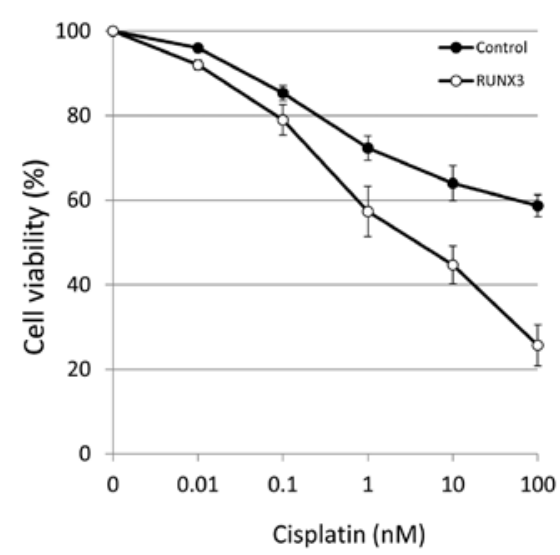

Huh7

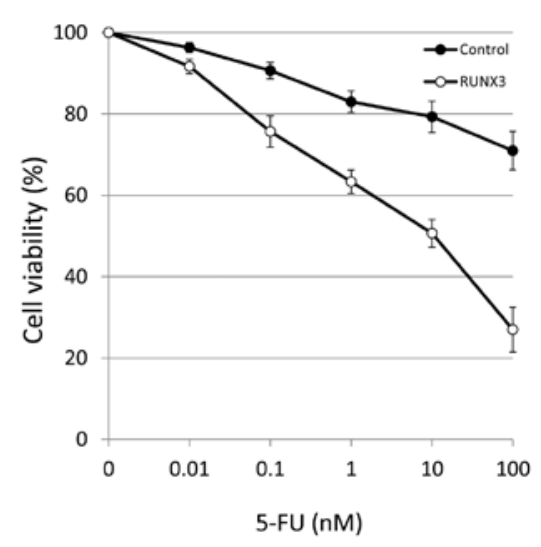

Huh7

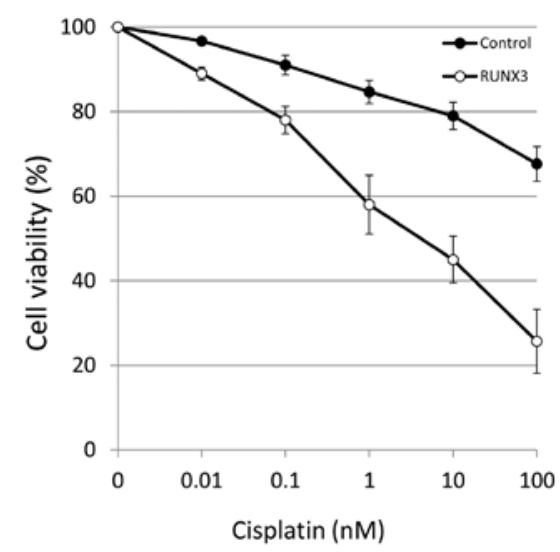

Figure 2. Cell growth activity and sensitivity to chemotherapeutic agents. Hep3B and Huh7 cells were treated with the indicated concentrations of 5-FU (A) and CDDP (B) for 3 days. Cell viability was measured by MTT assay. All results are expressed as ratios relative to the value on day 1 . Data represent the mean $\pm \mathrm{SE}$ of more than three independent experiments performed in triplicate.

A

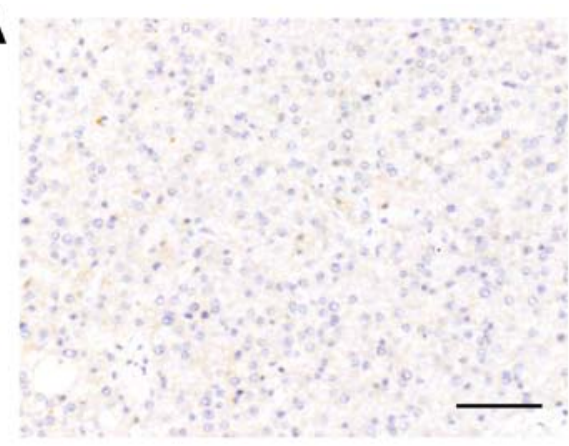

C

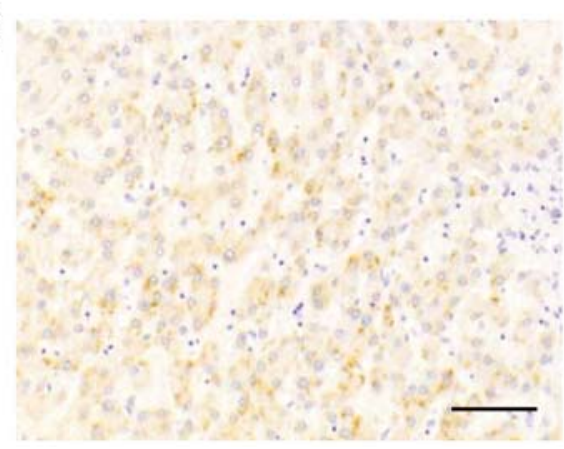

B

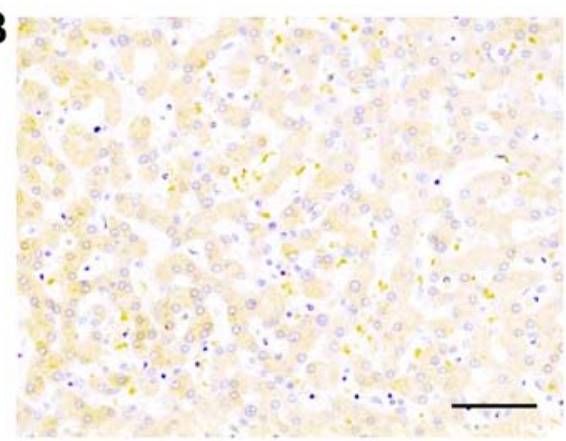

D

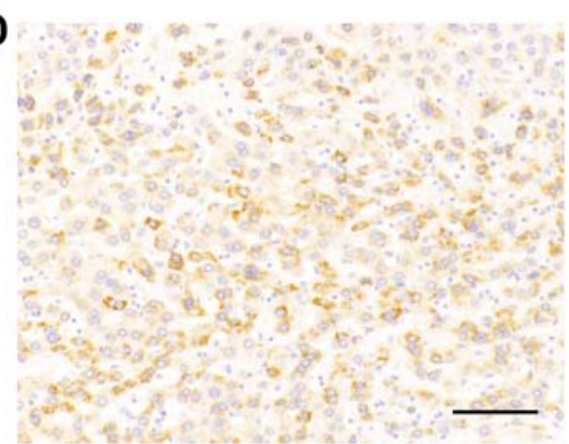

Figure 3. Immunohistochemical analysis of MRP expression. The images show the immunohistochemical staining of MRP1 (A, score of 0; B, score of 1; $\mathrm{C}$, score of 2; and D, score of 3). Scale bar, $100 \mu \mathrm{m}$. 
A

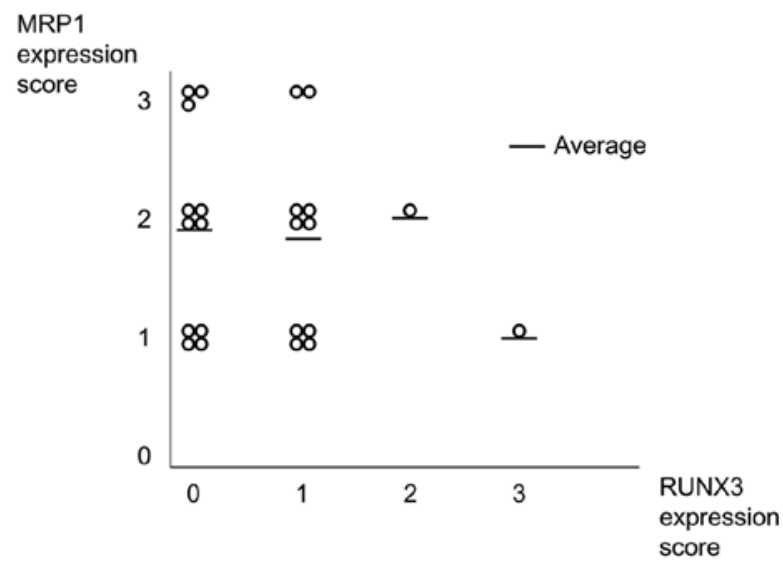

C

MRP3

expression

score

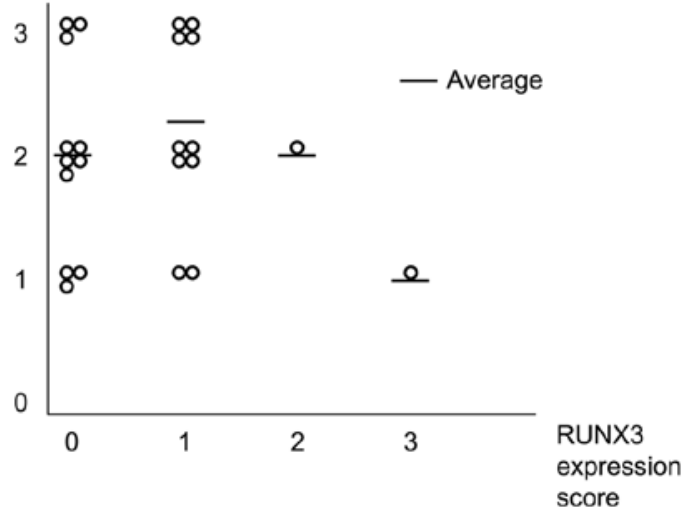

B

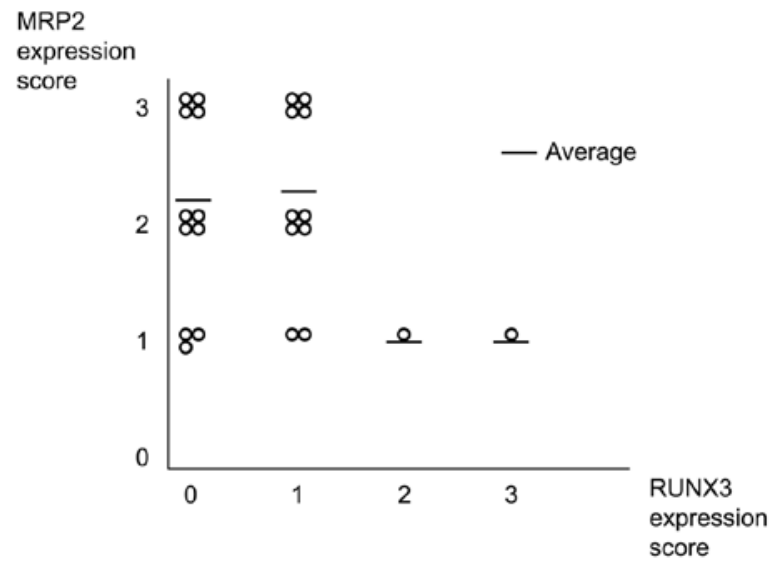

D

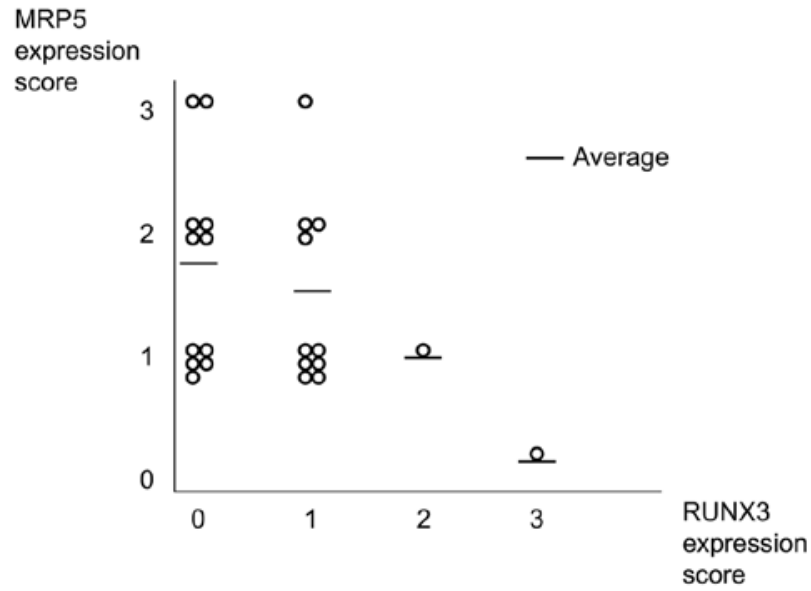

Figure 4. Correlation between RUNX3 expression and (A) MRP1, (B) MRP2, (C) MRP3 and (D) MRP5 expression scores in human HCC tissues. Plots of MRP expression scores are shown compared with RUNX3 expression scores.

MRP1

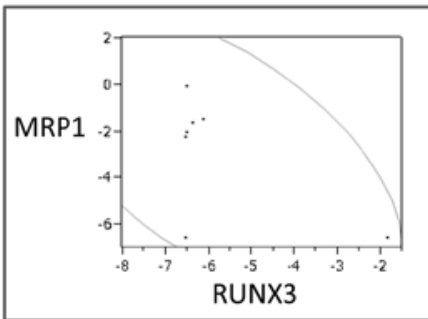

$r=-0.6007$

$P=0.1538$
MRP2

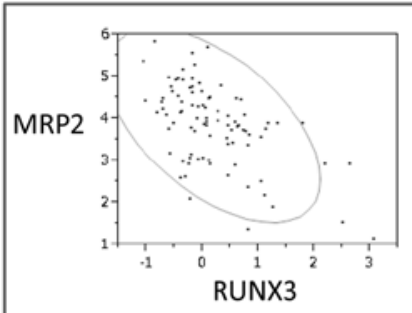

$r=-0.55729$

$P<0.0001$
MRP3

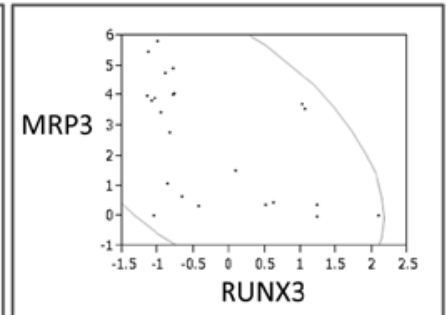

$r=-0.53741$

$P=0.0082$
MRP5

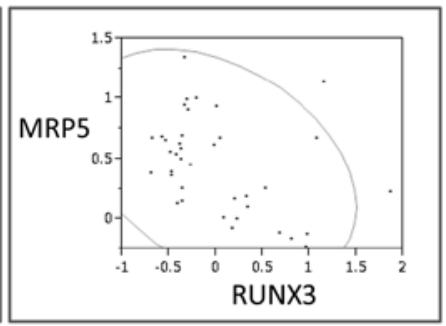

$\boldsymbol{r}=-0.36607$

$P=0.0238$

\section{RUNX3 expression}

Figure 5. Correlation between RUNX3 and MRP expression in human HCC data sets. The correlation between RUNX3 and MRP expression was analyzed using publically available microarray data sets (http://www.oncomine.org). The correlation coefficients and P-values of the relationships among RUNX3, MRP1, MRP2, MRP3 and MRP5 expression are shown. The 95\% tolerance ellipses for pairs of variables have been plotted. 


\section{Discussion}

Chemoresistance is a major challenge encountered during the therapeutic treatment of HCC. In the present study, we evaluated the relationship between RUNX3 expression and chemosensitivity in HCC. RUNX3 was found to act as a tumor suppressor in $\mathrm{HCC}$, consistent with previous studies showing that RUNX3 is frequently lost in HCC $(23,27-29)$ and that loss of RUNX3 contributes to the malignant transformation of HCC (23-25).

In a previous study of pancreatic cancer, we reported that the RUNX3 expression status affects gemcitabine sensitivity by attenuating MRP expression (26). Therefore, we hypothesized that RUNX3 expression may influence chemosensitivity in HCC. Drug resistance can be induced by several cellular processes. For example, MRPs mediate drug efflux, which decreases the accumulation of drugs within cancer cells. In the present study, we found that MRP expression generally decreased following ectopic expression of RUNX3 in all 3 HCC cell lines examined. Moreover, these cells exhibited higher chemosensitivity to 5-FU and CDDP than control cells, further supporting the role of MRPs in drug resistance in these cells.

Consistent with a previous study in pancreatic cancer, we found that RUNX3 blocked MRP expression in HCC cells. Moreover, MRP expression varied in HCC tissues and was positively correlated with RUNX3 protein expression. Since the number of HCC tissues in the present study was relatively small, we also assessed the relationship between MRPs and RUNX3 expression using the Oncomine database; this analysis further supported the positive correlation between RUNX3 and MRP expression. These results indicated that RUNX3 expression may regulate MRP expression. Notably, RUNX3 expression has been shown to sensitize gastric cancer cells to chemotherapeutic drugs by downregulating MRP1 through inhibition of MRP1 promoter activity (30). Thus, our findings suggest that RUNX3 may regulate MRP expression by inhibiting promoter activity in HCC cells and that targeting of RUNX3 and related signaling molecules may be a potential treatment modality for HCC.

Although we assessed the relationship between MRPs and RUNX3 expression in the present study, other factors have also been implicated in 5-FU and CDDP resistance. Multidrug resistance 1 (MDR1/ABCB1) and other $\mathrm{ABCB}$ transporters are also involved in the resistance against 5-FU $(10,31,32)$. CDDP resistance is established via a multifactorial mechanism including drug efflux and drug inactivation (33-35). In the present study, we did not evaluate the effects of RUNX3 protein expression on these mechanisms; thus, further studies are necessary.

In conclusion, the loss of RUNX3 expression may upregulate MRP expression, thereby contributing to 5-FU and CDDP resistance in patients with HCC. Moreover, re-expression of RUNX3 downregulated MRP expression and resensitized $\mathrm{HCC}$ cells to 5-FU and CDDP.

\section{Acknowledgements}

The authors thank Shin-ichi Nishina and Minoru Matsubara for their valuable suggestions.

\section{References}

1. El-Serag HB and Rudolph KL: Hepatocellular carcinoma: Epidemiology and molecular carcinogenesis. Gastroenterology 132: 2557-2576, 2007.

2. El-Serag HB: Hepatocellular carcinoma. N Engl J Med 365: 1118-1127, 2011.

3. Arumugam T, Ramachandran V, Fournier KF, Wang $\mathrm{H}$, Marquis L, Abbruzzese JL, Gallick GE, Logsdon CD, McConkey DJ and Choi W: Epithelial to mesenchymal transition contributes to drug resistance in pancreatic cancer. Cancer Res 69: 5820-5828, 2009.

4. Cheng L, Luo S, Jin C, Ma H, Zhou H and Jia L: FUT family mediates the multidrug resistance of human hepatocellular carcinoma via the PI3K/Akt signaling pathway. Cell Death Dis 4: e923, 2013.

5. Marin JJ, Monte MJ, Blazquez AG, Macias RI, Serrano MA and Briz O: The role of reduced intracellular concentrations of active drugs in the lack of response to anticancer chemotherapy. Acta Pharmacol Sin 35: 1-10, 2014.

6. Dean M, Hamon Y and Chimini G: The human ATP-binding cassette (ABC) transporter superfamily. J Lipid Res 42: 1007-1017, 2001.

7. Hagmann W, Jesnowski R, Faissner R, Guo C and Löhr JM: ATP-binding cassette $\mathrm{C}$ transporters in human pancreatic carcinoma cell lines. Upregulation in 5-fluorouracil-resistant cells. Pancreatology 9: 136-144, 2009.

8. Nambaru PK, Hübner T, Köck K, Mews S, Grube M, Payen L, Guitton J, Sendler M, Jedlitschky G, Rimmbach C, et al: Drug efflux transporter multidrug resistance-associated protein 5 affects sensitivity of pancreatic cancer cell lines to the nucleoside anticancer drug 5-fluorouracil. Drug Metab Dispos 39: 132-139, 2011.

9. Hagmann W, Faissner R, Schnölzer M, Löhr M and Jesnowski R: Membrane drug transporters and chemoresistance in human pancreatic carcinoma. Cancers 3: 106-125, 2010.

10. Hlavata I, Mohelnikova-Duchonova B, Vaclavikova R, Liska V, Pitule P, Novak P, Bruha J, Vycital O, Holubec L, Treska V, et al: The role of ABC transporters in progression and clinical outcome of colorectal cancer. Mutagenesis 27: 187-196, 2012.

11. Long J, Zhang Y, Yu X, Yang J, LeBrun DG, Chen C, Yao Q and Li M: Overcoming drug resistance in pancreatic cancer. Expert Opin Ther Targets 15: 817-828, 2011.

12. Wang WB, Yang Y, Zhao YP, Zhang TP, Liao Q and Shu H: Recent studies of 5-fluorouracil resistance in pancreatic cancer. World J Gastroenterol 20: 15682-15690, 2014.

13. Park JY, Ahn SH, Yoon YJ, Kim JK, Lee HW, Lee Y, Chon CY, Moon YM and Han KH: Repetitive short-course hepatic arterial infusion chemotherapy with high-dose 5-fluorouracil and cisplatin in patients with advanced hepatocellular carcinoma. Cancer 110: 129-137, 2007.

14. Ma MC, Chen YY, Li SH, Cheng YF, Wang CC, Chiu TJ, Pei SN, Liu CT, Huang TL, Huang CH, et al: Intra-arterial chemotherapy with doxorubicin and cisplatin is effective for advanced hepatocellular cell carcinoma. ScientificWorldJournal 2014: 160138, 2014.

15. Johnson PJ: Are there indications for chemotherapy in hepatocellular carcinoma? Surg Oncol Clin N Am 12: 127-134, 2003.

16. Nishimura M: A successful treatment by hepatic arterial infusion therapy for advanced, unresectable biliary tract cancer. World J Hepatol 2: 192-197, 2010.

17. Shen DW, Pouliot LM, Hall MD and Gottesman MM: Cisplatin resistance: A cellular self-defense mechanism resulting from multiple epigenetic and genetic changes. Pharmacol Rev 64: 706-721, 2012.

18. Kowalski P, Surowiak P and Lage H: Reversal of different drug-resistant phenotypes by an autocatalytic multitarget multiribozyme directed against the transcripts of the $\mathrm{ABC}$ transporters MDR1/P-gp, MRP2, and BCRP. Mol Ther 11: 508-522, 2005.

19. Kuwano M, Toh S, Uchiumi T, Takano H, Kohno K and Wada M: Multidrug resistance-associated protein subfamily transporters and drug resistance. Anticancer Drug Des 14: 123-131, 1999.

20. Materna V, Holm PS, Dietel $M$ and Lage H: Kinetic characterization of ribozymes directed against the cisplatin resistance-associated ABC transporter cMOAT/MRP2/ABCC2. Cancer Gene Ther 8: 176-184, 2001.

21. Li QL, Ito K, Sakakura C, Fukamachi H, Inoue K, Chi XZ, Lee KY, Nomura S, Lee CW, Han SB, et al: Causal relationship between the loss of $R U N X 3$ expression and gastric cancer. Cell 109: 113-124, 2002. 
22. Subramaniam MM, Chan JY, Yeoh KG, Quek T, Ito $\mathrm{K}$ and Salto-Tellez M: Molecular pathology of RUNX3 in human carcinogenesis. Biochim Biophys Acta 1796: 315-331, 2009.

23. Nakanishi Y, Shiraha H, Nishina S, Tanaka S, Matsubara M, Horiguchi S, Iwamuro M, Takaoka N, Uemura M, Kuwaki K, et al: Loss of runt-related transcription factor 3 expression leads hepatocellular carcinoma cells to escape apoptosis. BMC Cancer 11: 3, 2011.

24. Nishina S, Shiraha H, Nakanishi Y, Tanaka S, Matsubara M, Takaoka N, Uemura M, Horiguchi S, Kataoka J, Iwamuro M, et al: Restored expression of the tumor suppressor gene RUNX3 reduces cancer stem cells in hepatocellular carcinoma by suppressing Jagged1-Notch signaling. Oncol Rep 26: 523-531, 2011.

25. Tanaka S, Shiraha H, Nakanishi Y, Nishina S, Matsubara M, Horiguchi S, Takaoka N, Iwamuro M, Kataoka J, Kuwaki K, et al: Runt-related transcription factor 3 reverses epithelialmesenchymal transition in hepatocellular carcinoma. Int $\mathrm{J}$ Cancer 131: 2537-2546, 2012.

26. Horiguchi S, Shiraha H, Nagahara T, Kataoka J, Iwamuro M, Matsubara M, Nishina S, Kato H, Takaki A, Nouso K, et al: Loss of runt-related transcription factor 3 induces gemcitabine resistance in pancreatic cancer. Mol Oncol 7: 840-849, 2013

27. Mori T, Nomoto S, Koshikawa K, Fujii T, Sakai M, Nishikawa Y, Inoue S, Takeda S, Kaneko T and Nakao A: Decreased expression and frequent allelic inactivation of the RUNX3 gene at $1 \mathrm{p} 36$ in human hepatocellular carcinoma. Liver Int 25: 380-388, 2005.

28. Xiao WH and Liu WW: Hemizygous deletion and hypermethylation of RUNX3 gene in hepatocellular carcinoma. World $\mathrm{J}$ Gastroenterol 10: 376-380, 2004.
29. Li X, Zhang Y, Zhang Y, Qiao T, Wu K, Ding J, Liu J and Fan D: RUNX3 inhibits growth of HCC cells and HCC xenografts in mice in combination with adriamycin. Cancer Biol Ther 7: 669-676, 2008.

30. Guo C, Ding J, Yao L, Sun L, Lin T, Song Y, Sun L and Fan D Tumor suppressor gene Runx3 sensitizes gastric cancer cells to chemotherapeutic drugs by downregulating Bcl-2, MDR-1 and MRP-1. Int J Cancer 116: 155-160, 2005.

31. Lu F, Hou YQ, Song Y and Yuan ZJ: TFPI-2 downregulates multidrug resistance protein in 5-FU-resistant human hepatocellular carcinoma BEL-7402/5-FU cells. Anat Rec 296: 56-63, 2013.

32. Gu W, Fang FF, Li B, Cheng BB and Ling CQ: Characterization and resistance mechanisms of a 5-fluorouracil-resistant hepatocellular carcinoma cell line. Asian Pac J Cancer Prev 13: 4807-4814, 2012.

33. Zisowsky J, Koegel S, Leyers S, Devarakonda K, Kassack MU, Osmak M and Jaehde U: Relevance of drug uptake and efflux for cisplatin sensitivity of tumor cells. Biochem Pharmacol 73: 298-307, 2007.

34. Siddik ZH: Cisplatin: Mode of cytotoxic action and molecular basis of resistance. Oncogene 22: 7265-7279, 2003.

35. Samimi G, Katano K, Holzer AK, Safaei R and Howell SB: Modulation of the cellular pharmacology of cisplatin and its analogs by the copper exporters ATP7A and ATP7B. Mol Pharmacol 66: 25-32, 2004. 